\title{
Nodal-line semimetal HMTSF-TCNQ: Anomalous orbital diamagnetism and charge density wave
}

\author{
S. Ozaki, ${ }^{1, *}$ I. Tateishi, ${ }^{1,2}$ H. Matsuura, ${ }^{1}$ M. Ogata, ${ }^{1,3}$ and K. Hiraki ${ }^{4,5}$ \\ ${ }^{1}$ Department of Physics, University of Tokyo, Bunkyo, Tokyo 113-0033, Japan \\ ${ }^{2}$ RIKEN Center for Emergent Matter Science, Wako, Saitama 351-0198, Japan \\ ${ }^{3}$ Trans-scale Quantum Science Institute, University of Tokyo, Bunkyo, Tokyo 113-0033, Japan \\ ${ }^{4}$ Fukushima Medical University, Fukushima 960-1295, Japan \\ ${ }^{5}$ IMR, Tohoku University, Miyagi 980-8577, Japan
}

(Dated: June 9, 2021)

\begin{abstract}
This study investigates the electronic states and physical quantities of an organic charge-transfer complex HMTSF-TCNQ, which undergoes a charge-density-wave (CDW) phase transition at temperature $T_{c} \simeq 30 \mathrm{~K}$. A first-principles calculation is utilized to determine that the normal state is a topological semimetal with open nodal lines. Besed on the first-principles calculation, we develop a tight-binding model to investigate the electronic state in detail. Below $T_{c}$, the CDW phase is examined in the tight-binding scheme using the mean-field approximation. It is shown that the open nodal lines are deformed into closed ones, and their shapes are sensitive to the order parameter. Using this tight-binding model, we theoretically evaluate the temperature dependencies of two physical quantities: the spin-lattice relaxation time $T_{1}$ and the orbital magnetic susceptibility. In particular, an anomalous plateau is obtained at low temperatures in the orbital diamagnetism. We presume that this anomalous plateau originates owing to the conflict between the interband diamagnetism, impurity scattering, and the nodal line deformation. We also conduct an experiment to investigate the orbital magnetism, and the results are in excellent quantitative agreement with the theory.
\end{abstract}

\section{INTRODUCTION}

A charge density wave $(\mathrm{CDW})$ in topological bands induces fascinating phenomena, such as the threedimensional Hall effect [1] and axionic CDW phase [2], which have recently generated considerable research interest. A CDW is a quantum phase that is typical of quasi-one-dimensional organic conductors, and the topological properties of organic materials have become more extensively researched in the last two decades [3-11]. Organic materials that possess topological properties have the potential for novel phenomena that originate from the interplay between their topological bands and the CDW. Such materials require both one-dimensionality for the CDW and two- or three-dimensionality for the topological nature. One example of these materials is HMTSF-TCNQ (hexamethylene-tetraselena-fulvalenetetracyanoquindimethane) [12-15].

HMTSF-TCNQ is a classical quasi-one-dimensional charge-transfer complex that was discovered in the 1970s, and it has attracted considerable attention in contemporary research, owing to the possibility of field-induced CDW [16-18]. HMTSF-TCNQ has components that are similar to those of the well-known organic complexes TTF-TCNQ and $\mathrm{TMTSF}_{2} \mathrm{PF}_{6}$, which are known as a typical organic conductor that has a CDW and the first organic superconductor identified, respectively [19, 20]. These two materials have been studied extensively; however, HMTSF-TCNQ has not.

HMTSF-TCNQ undergoes a CDW transition at approximately $30 \mathrm{~K}$ under ambient pressure; however, the

* ozaki@hosi.phys.s.u-tokyo.ac.jp temperature dependence of the resistivity is different from that of TTF-TCNQ in that a clear metal-insulator transition is not observed. According to Refs. [15, 19], the Fermi surface of HMTSF-TCNQ is two-dimensional, owing to the relatively large interchain hoppings, which causes incomplete CDW nesting. In this material, unconventional temperature dependencies of physical quantities, such as the magnetic susceptibility [14], Seebeck coefficient [12], and the electric conductivity are known and are presumed to be attributed to the incomplete CDW nesting.

Despite these intriguing properties, there are no reliable theoretical models for the electronic states of the normal phase or the CDW phase. The electronic state of the normal phase is typically discussed in terms of Weger's model [15], which is a four-band tight-binding model that consists of HOMOs (highest occupied molecular orbitals) and LUMOs (lowest unoccupied molecular orbitals) for each molecule on the basis of the crystal structure known at that time with the estimated hopping parameters. This model was able to explain some experimental results; however, a subsequent experimental study suggested the crystal structure of a different space group [21]. Furthermore, the first-principles calculation based on the new crystal structure, which is presented in this study, suggests Fermi surfaces of different shapes and locations from those calculated by Weger. Therefore, we need to modify the existing model on the basis of these results.

The physical quantities of HMTSF-TCNQ are of interest as well as its electronic states. In particular, HMTSFTCNQ shows a large diamagnetism at low temperatures, and it reaches an anomalous plateau below $T_{c}$ [14]. Weger tried to explain this behavior via the model and con- 
cluded that the diamagnetism was attributed to Landau diamagnetism. However, this claim is debatable because Landau diamagnetism is derived from the electrons on the Fermi surface. In the CDW state, the density of states (DOS) at the Fermi level is expected to be small. Therefore, Landau diamagnetism does not explain the large diamagnetism at low temperatures; thus, the origin of the diamagnetism remains unknown.

To address this problem, the current study serves a twofold purpose. One is to reveal the electronic states of the normal and CDW phases of HMTSF-TCNQ under ambient pressure. First, we implement a first-principles calculation based on the correct crystal structure. The obtained results, in particular the symmetry indicator, indicate that its normal phase is a topologically protected nodal line semimetal. On the basis of these results, we construct a tight-binding model and discuss the nodal lines in detail. These analyses clarify that the energy at the node points fluctuate along the nodal lines, which results in electron and hole pockets. As a result, we expect that the nodal lines influence various physical quantities that are susceptible to the Fermi surface. Furthermore, assuming a plausible CDW nesting, we discuss how the CDW affects the nodal lines and the electron and hole pockets. We theoretically evaluate the spin-lattice relaxation time $T_{1}$ for the experimental confirmation of the existence of the nodal lines.

The other objective is to clarify the derivation of the large diamagnetism and its temperature dependence. Since the nodal lines are located near the Fermi level, a large diamagnetism derived from the interband effect is strongly expected from an analogy to the case of the Dirac electrons [22-28]. Note that the interband effect is insensitive to the DOS at the Fermi level. Using the Fukuyama formula [29], which properly describes the interband effect, we evaluate the magnetic susceptibility. An experiment is conducted to determine the magnetic susceptibility using a superconducting quantum interference device (SQUID) magnetometer. The proposed theory is in excellent agreement with this experimental result.

This paper is organized as follows. In Sec. II, the correct crystal structure of HMTSF-TCNQ is presented. In Sec. III, we investigate the electronic state of HMTSFTCNQ using the first-principles calculation, and we demonstrate the existence of nodal lines. In Sec. IV, we construct a tight-binding model based on the results of the previous section and discuss the nodal lines in detail. In Sec. V, we introduce the CDW with the mean-field approximation and examine the CDW phase. In Sec. VI, we evaluate the spin-lattice relaxation time $T_{1}$ and the orbital magnetic susceptibility. A comparison between the theory and the magnetic susceptibility experimental results is also conducted. Finally, we present the conclusion to the study in Sec. VII.

\section{CRYSTAL STRUCTURE OF HMTSF-TCNQ}

HMTSF-TCNQ consists of two organic molecules, HMTSF and TCNQ, the structure of which are shown in Fig. 1(a). This material has a base-centered monoclinic lattice, and for convenience, we introduce two different notations of the unit cell: the conventional cell and the primitive cell, which are shown in Figs. 1(b) and $1(\mathrm{c})$, respectively. The crystal structures and wave functions in this study are drawn using VESTA (JP minerals, Japan) [30]. Each molecule forms a one-dimensional chain, and the HMTSF and TCNQ chains are arranged in a checkerboard configuration, as can be observed in Fig. 1(b). Throughout this study, we use the Cartesian coordinate system, as shown in Fig. 2(a) (the unique axis $\boldsymbol{b}$ convention). The space group is $C 2 / m$ (No. 12), the generators of which are lattice translations, $C_{2 y}$ rotation, and inversion $I$ [31]. Recent measurements for high quality samples have determined the lattice constants and atomic coordinates at room temperature [32], which are used in this study. The experimental details will be presented elsewhere. The newly obtained lattice constants are slightly different from those presented in a previous study [21]. These values are tabulated in Table I.

Figure 2(a) shows the basic lattice vectors for the primitive cell, $\boldsymbol{a}_{1}=(a / 2, b / 2,0), \boldsymbol{a}_{2}=(-a / 2, b / 2,0)$, and $\boldsymbol{a}_{3}=(c \cos \beta, 0, c \sin \beta)$. The orientation of the chains are parallel to $\boldsymbol{a}_{3}$, and the corresponding reciprocal lattice vectors are $\boldsymbol{b}_{1}=2 \pi(1 / a, 1 / b,-1 / a \tan \beta), \boldsymbol{b}_{2}=$ $2 \pi(-1 / a, 1 / b, 1 / a \tan \beta)$, and $\boldsymbol{b}_{3}=2 \pi(0,0,1 / c \sin \beta)$. Note that $\boldsymbol{a}_{3}$ is almost parallel to the $z$ axis, but slightly inclined towards the negative $x$ direction and that $\boldsymbol{b}_{3}$ is parallel to the $k_{z}$ axis. Figure 2(b) shows the first Brillouin zone, which is a hexagonal column, and the timereversal invariant momenta (TRIM) are $\Gamma, \mathrm{A}, \mathrm{Y}, \mathrm{M}$, two $\mathrm{V}$, and two L points.

TABLE I. Lattice constants for the conventional cell.

\begin{tabular}{ccccc}
\hline \hline & $a / \AA$ & $b / \AA$ & $c / \AA$ & $\beta /{ }^{\circ}$ \\
\hline Ref. [21] & $21.999(14)$ & 12.573 & $3.890(1)$ & $90.29(4)$ \\
present [32] & 21.85 & 12.48 & 3.87 & 90.25 \\
\hline \hline
\end{tabular}

\section{FIRST-PRINCIPLES CALCULATION OF HMTSF-TCNQ}

In this section, we present the electronic state of HMTSF-TCNQ, obtained by a first-principles calculation. This calculation is performed by QUANTUM ESPRESSO [33], which uses the density functional theory $[34,35]$. We neglect spin-orbit interaction because in organic materials, it is typically negligible. For the exchange-correlation term, the generalized gradient approximation with nonrelativistic Perdew-BurkeErnzerhof parametrization [36] is used. The Kohn-Sham 
(a)<smiles></smiles><smiles>N#CC(C#N)=c1ccc(=C(C#N)C#N)cc1</smiles>

(b)
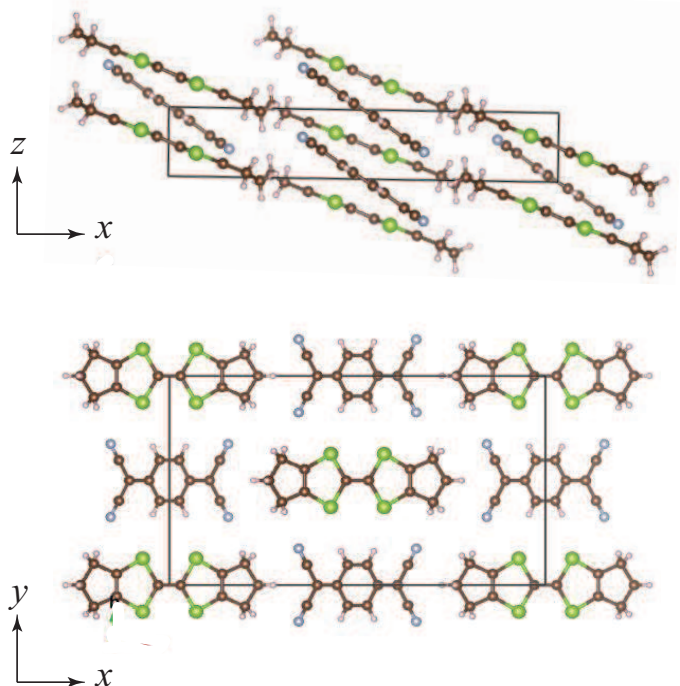

(c)
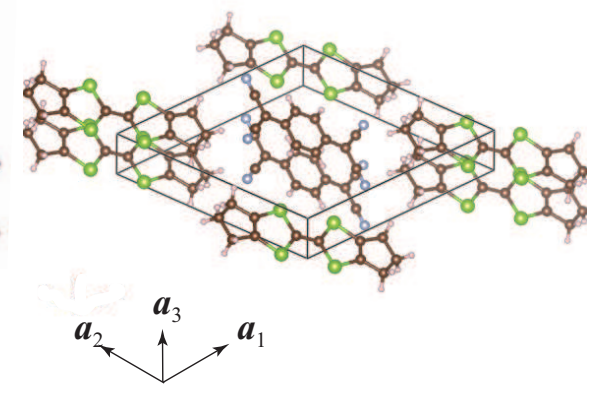

FIG. 1. (a) Molecular structures of HMTSF and TCNQ. (b) Conventional and (c) primitive cell of HMTSF-TCNQ. Brown, green, blue, and white circles represent $\mathrm{C}$, Se, N, and $\mathrm{H}$ atoms, respectively. The black lines represent the boundary of each unit cell. The primitive cell is half the size of the conventional cell.

(a)

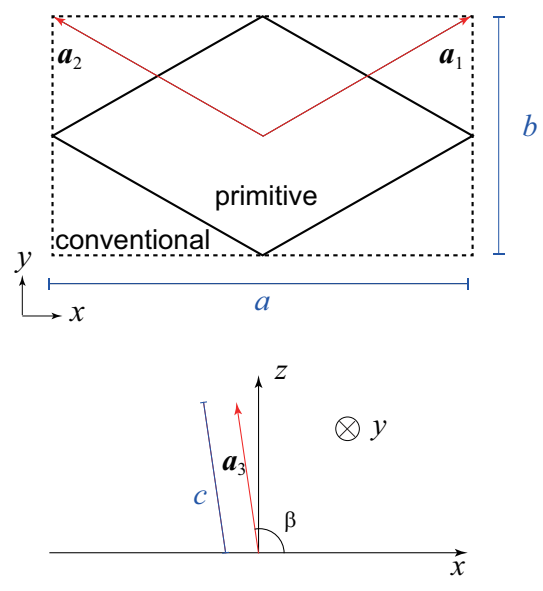

(b)

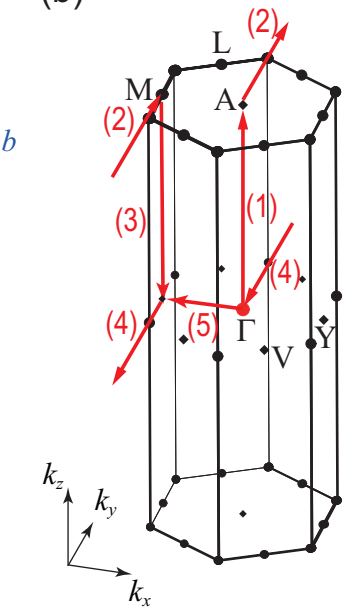

FIG. 2. (a) Basic lattice vectors for the primitive cell of HMTSF-TCNQ. The rhombus and dashed rectangle represent the boundary of the primitive and conventional cell, respectively. The lattice constants $a, b, c$, and $\beta$ are for the conventional cell. (b) First Brillouin zone and TRIM. The TRIM are $\Gamma, A, Y, M$, two $\mathrm{V}$, and two L points. The path for the energy dispersion is also shown.

orbitals are expanded with plane waves and the cutoff energies are 70 and $320 \mathrm{Ry}$ for wave functions and charge density, respectively. The $\boldsymbol{k}$-point grid on the Brillouin zone is taken as $8 \times 8 \times 40$.

The obtained energy dispersion and DOS are shown in Fig. 3(a), and the correspoding path is shown in

Fig. 2(b). First, we find that the dispersion is almost flat in the $k_{x}$ and $k_{y}$ directions, and the DOS has peaks at the band edges. These properties are characteristic of quasi-one-dimensional materials. Another important feature is that the two bands near the Fermi level are very isolated from other bands. This implies that the mixing of other orbitals is small and that it is sufficient to consider these two bands to discuss the low-energy properties of this material. An energy gap is observed in the energy dispersion at the Fermi energy in Fig. 3(a); however, the DOS does not show a clear gap. As shown below, this is because there are nodal lines away from the high-symmetry $\boldsymbol{k}$ points. This Dirac-type dispersion may strongly affect physical quantities, such as the orbital diamagnetism.

Next, we examine the spatial distribution of the wave functions. Figures 3(b) and 3(c) show the wave functions at the $\Gamma$ point for the valence and conduction bands, respectively. We observe that the wave functions at other high-symmetry points also consist of these two Wannier functions. (The wave functions at other high-symmetry points are shown in Appendix A.) Therefore, we can naturally assume that the two bands at each $\boldsymbol{k}$ point are well described by the linear combination of these two Wannier functions.

Thirdly, we discuss the topological nature of the material using the symmetry indicator. We can observe that there is no band crossing on the high-symmetry lines or planes. However, the space group No. 12 can have nodal lines at generic points [37-40]. The existence of nodal lines is diagnosed by the symmetry indicator $\left(z_{2,2}, z_{2}^{\prime}\right)$, 
(a)

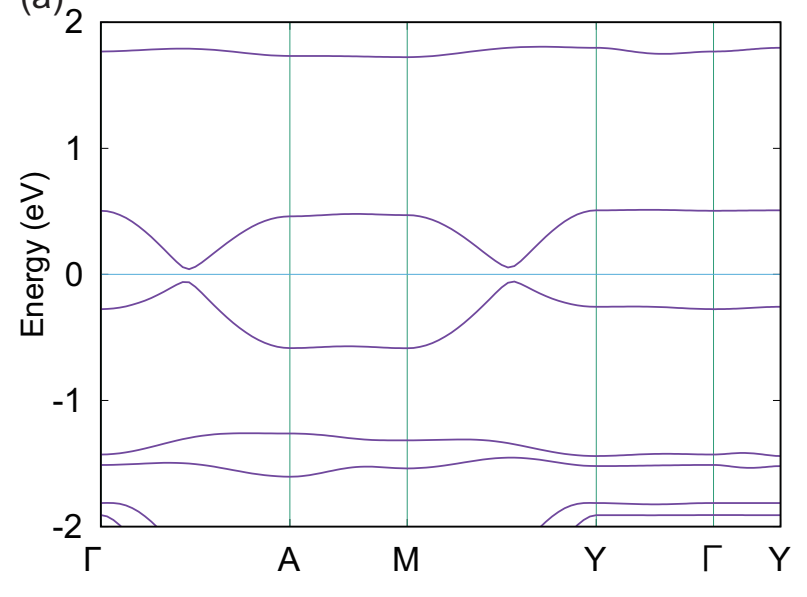

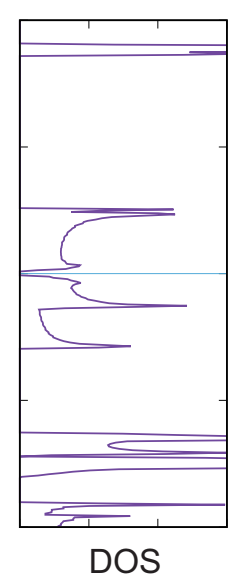

(b)

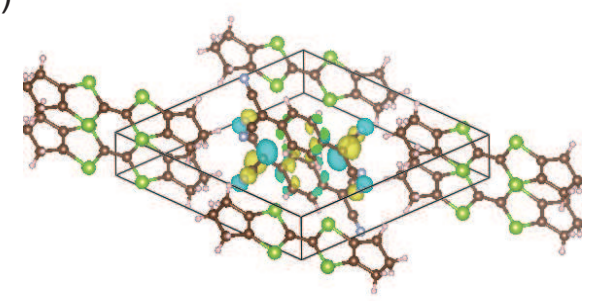

(c)

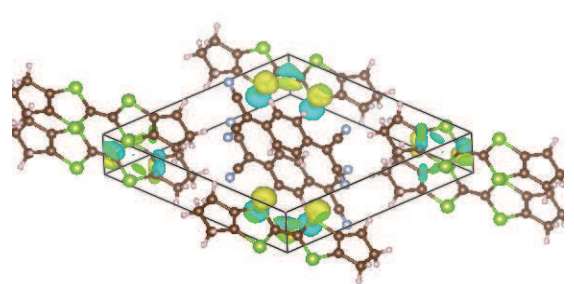

FIG. 3. (a) Energy dispersion and DOS of HMTSF-TCNQ. Wave functions for (b) valence and (c) conduction bands at the $\Gamma$ point. These wave functions consist of two Wannier functions, which are the same for wave functions at other high-symmetry points, shown in Appendix A.

the components of which are defined by

$$
\begin{aligned}
z_{2,2} & =\sum_{\boldsymbol{k}=\mathrm{V}, \mathrm{Y}, \mathrm{M}, \mathrm{L}} n^{-}(\boldsymbol{k})(\bmod 2), \\
z_{2}^{\prime} & =\frac{1}{2} \sum_{\boldsymbol{k}=8 \mathrm{TRIM}} n^{-}(\boldsymbol{k})(\bmod 2),
\end{aligned}
$$

where $n^{-}(\boldsymbol{k})$ is the number of occupied bands with odd parity at $\boldsymbol{k}$. This indicator is calculated by examining the parities of the wave functions, and we obtain a nontrivial indicator $\left(z_{2,2}, z_{2}^{\prime}\right)=(1,1)$. This value indicates the existence of open nodal lines approximately along the $k_{x}$ direction [41]. (The parities at TRIM are shown in Appendix A.)

\section{TIGHT-BINDING MODEL AND NODAL LINES}

\section{A. Tight-binding model}

In the previous section, the discussion based on the symmetry indicator suggests the existence of the nodal lines. However, the symmetry indicator does not indicate their exact locations or detailed properties. Therefore, we construct a tight-binding model and analyze it to clarify the detailed electronic properties.

As shown in the previous section, the wave functions near the Fermi level consist of the two Wannier functions located in HMTSF and TCNQ molecules. From these orbitals, we construct a two-band tight-binding model using Slater-Koster's method [42]. We fit the hopping parameters to reproduce the band dispersion using WANNIER90 [43]. From this result, we consider the eight largest hopping parameters and neglect the others. The chosen hoppings are shown in Fig. 4, and the fitted parameters are summarized in Table II. We measure the energies from the Fermi level (i.e., $\mu=0$ ) and denote the one-body level for the HMTSF (TCNQ) orbital as $\varepsilon_{\mathrm{H}}$ $\left(\varepsilon_{\mathrm{T}}\right)$. Using the Fourier transform, we obtain the twoband tight-binding model in the $\boldsymbol{k}$ space as follows:

$$
H_{0}(\boldsymbol{k})=\left(\begin{array}{cc}
t_{\mathrm{HH}}(\boldsymbol{k}) & t_{\mathrm{HT}}^{*}(\boldsymbol{k}) \\
t_{\mathrm{HT}}(\boldsymbol{k}) & t_{\mathrm{TT}}(\boldsymbol{k})
\end{array}\right),
$$

where

$t_{\mathrm{HH}}(\boldsymbol{k})=\varepsilon_{\mathrm{H}}+2 t_{\mathrm{HH}}^{z} \cos \tilde{k}_{z} c+2 t_{\mathrm{HH} 2}^{z} \cos 2 \tilde{k}_{z} c+2 t_{\mathrm{HH}}^{y} \cos k_{y} b$,

$$
t_{\mathrm{TT}}(\boldsymbol{k})=\varepsilon_{\mathrm{T}}+2 t_{\mathrm{TT}}^{z} \cos \tilde{k}_{z} c+2 t_{\mathrm{TT} 2}^{z} \cos 2 \tilde{k}_{z} c+2 t_{\mathrm{TT}}^{y} \cos k_{y} b,
$$

$$
\begin{aligned}
t_{\mathrm{HT}}(\boldsymbol{k})= & 4 i t_{\mathrm{HT}}^{y} \cos \frac{k_{y} b}{2} \sin \frac{\tilde{k}_{z} c}{2}+2 i t_{\mathrm{HT} 1}^{x} \sin \left(-\frac{k_{x} a}{2}+\frac{\tilde{k}_{z} c}{2}\right) \\
& +2 i t_{\mathrm{HT} 2}^{x} \sin \left(\frac{k_{x} a}{2}+\frac{\tilde{k}_{z} c}{2}\right)
\end{aligned}
$$

with $\tilde{k}_{z}=k_{x} \cos \beta+k_{z} \sin \beta$.

Diagonalizing the Hamiltonian in Eq. (3), we obtain the energy dispersion:

$E_{ \pm}=\frac{t_{\mathrm{HH}}(\boldsymbol{k})+t_{\mathrm{TT}}(\boldsymbol{k})}{2} \pm \sqrt{\left|t_{\mathrm{HT}}(\boldsymbol{k})\right|^{2}+\frac{1}{4}\left(t_{\mathrm{HH}}(\boldsymbol{k})-t_{\mathrm{TT}}(\boldsymbol{k})\right)^{2}}$,

which is shown in Fig. 5(a). The total DOS is shown in Fig. 5(b). These results accurately reproduce those by the first-principles calculation shown in Fig. 3(a). In the DOS, we observe a linear behavior near the Fermi level, 

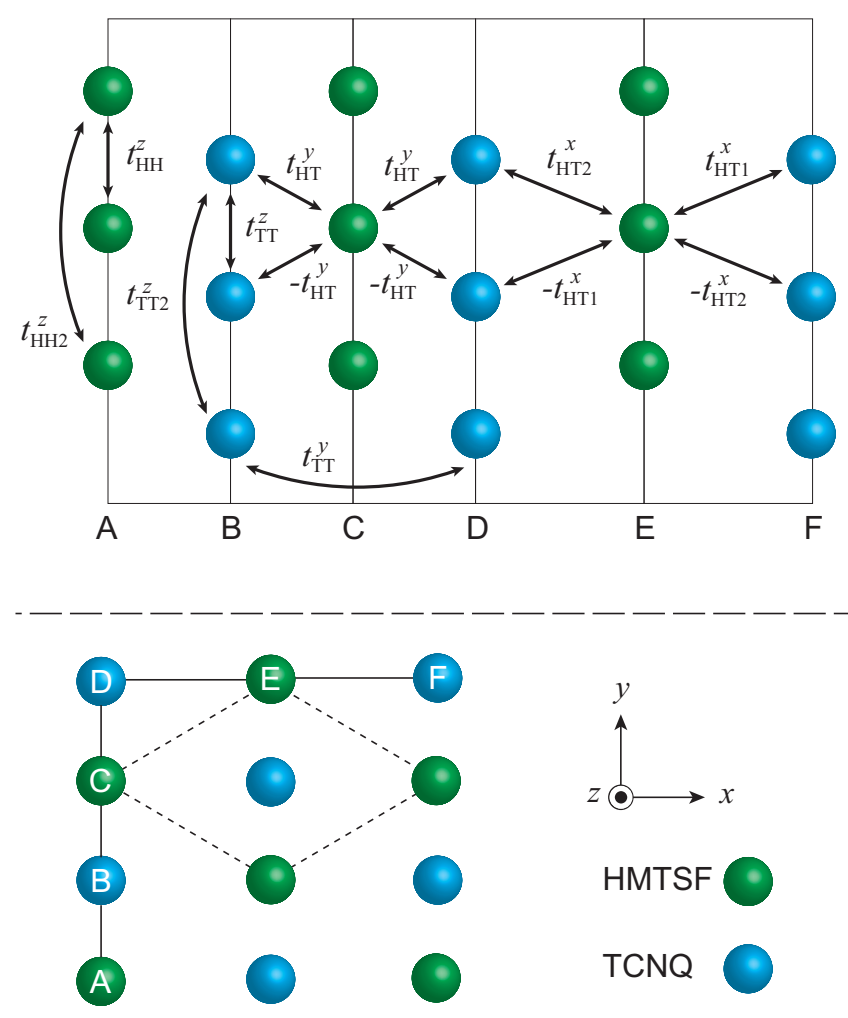

FIG. 4. Eight largest hopping parameters used in the tightbinding model [Eq. (6)]. The subscript and superscript represent the related molecules and the direction of the hopping, respectively. The lower panel shows the configuration of the molecules viewed from the $z$ direction, and $\mathrm{A}-\mathrm{F}$ indicate the positions of atoms in the upper panel. The dashed line represents the primitive unit cell.

which is typical for two-dimensional Dirac electrons. Figure 5(c) shows the partial DOS for HMTSF and TCNQ molecules. While these wave functions decouple at the band edge, they contribute almost equally to the DOS at the Fermi level. This result supports the existence of the Dirac-type dispersion, because it always consists of at least two orbitals. The shape of the Dirac cone calculated from the proposed tight-binding model is shown in

TABLE II. Hopping parameteres between Wannier functions.

\begin{tabular}{cc}
\hline \hline Hopping parameters & Energy / eV \\
\hline$t_{\mathrm{HH}}^{z}$ & 0.269 \\
$t_{\mathrm{TT}}^{z}$ & -0.184 \\
$t_{\mathrm{HH} 2}^{z}$ & 0.0106 \\
$t_{\mathrm{TT} 2}^{z}$ & 0.0126 \\
$t_{\mathrm{TT}}^{y}$ & -0.00358 \\
$t_{\mathrm{HT}}^{y}$ & 0.0193 \\
$t_{\mathrm{HT} 1}^{x}$ & -0.00689 \\
$t_{\mathrm{HT} 2}^{x}$ & 0.00446 \\
\hline$\varepsilon_{\mathrm{H}}$ & -0.0316 \\
$\varepsilon_{\mathrm{T}}$ & 0.0542 \\
\hline \hline
\end{tabular}

Appendix B.

\section{B. Nodal lines}

This subsection discusses the locations of the nodal lines in the $\boldsymbol{k}$ space. From the energy dispersion Eq. (7), we can identify the nodal lines by the following condition:

$$
t_{\mathrm{HT}}(\boldsymbol{k})=0, \quad t_{\mathrm{HH}}(\boldsymbol{k})=t_{\mathrm{TT}}(\boldsymbol{k}) .
$$

Solving Eq. (8) numerically, we obtain the configuration of the nodal lines as shown in Figs. 6(a) and 6(b). The bird's eye view [Fig. 6(a)] indicates that the nodal lines are approximately along the $k_{x}$ direction, as discussed in Sec. III, and are almost located on the planes perpendicular to the $k_{z}$ axis. Becuase the crystal has $C_{2 y}$ rotation, inversion, and $\sigma_{y}$ symmetries, the nodal lines have the same symmetries. In particular, owing to the $C_{2 y}$ rotation symmetry, the projection of the nodal lines onto the $k_{x}-k_{y}$ plane [Fig. 6(b)] intersect on the $k_{x}=0$ line. Figure 6(c) shows the Fermi surface of the proposed tight-binding model, where the purple and green surfaces represent the electron and hole pockets, respectively. The energy at the Dirac node point fluctuates along the nodal lines, which results in alternating thin tube-shaped electron and hole pockets.

\section{CDW PHASE}

HMTSF-TCNQ exhibits a CDW phase transition at $T_{c} \simeq 30 \mathrm{~K}$. Theoretically, the nesting vector of this CDW state is presumed to be the vector between Dirac points on the nodal lines at the Fermi level, as shown in Fig. 6(a). In the proposed model, the nesting vector is $\boldsymbol{q} \simeq(0,0,0.937 \pi / c \cos \beta)$. Although the obtained nesting vector is slightly different from the experimental value [44], $\boldsymbol{q} \simeq(0,0,0.74 \pi / c \cos \beta)$, we use the former in the following analyses.

Considering the nesting in the mean-field approximation [45-47], the effective model becomes

$H_{\mathrm{CDW}}(\boldsymbol{k})=\left(\begin{array}{cccc}t_{\mathrm{HH}}(\boldsymbol{k}) & t_{\mathrm{HT}}^{*}(\boldsymbol{k}) & \Delta & 0 \\ t_{\mathrm{HT}}(\boldsymbol{k}) & t_{\mathrm{TT}}(\boldsymbol{k}) & 0 & \Delta \\ \Delta & 0 & t_{\mathrm{HH}}(\boldsymbol{k}-\boldsymbol{q}) & t_{\mathrm{HT}}^{*}(\boldsymbol{k}-\boldsymbol{q}) \\ 0 & \Delta & t_{\mathrm{HT}}(\boldsymbol{k}-\boldsymbol{q}) & t_{\mathrm{TT}}(\boldsymbol{k}-\boldsymbol{q})\end{array}\right)$

where $\Delta$ is the order parameter of the CDW state, which is assumed to be common in the HMTSF and TCNQ chain for simplicity. Note that the periodicity in the $k_{z}$ direction is lost and that $k_{z}$ is only allowed in the vicinity of the nodal lines in the $k_{z}>0$ region $\left(k_{z} \simeq 0.38 \AA^{-1}\right)$. However, the nodal lines are located on almost the same plane as in the case of $\Delta=0$; therefore, we can still discuss the nodal lines using the effective Hamiltonian in Eq. (9).

Diagonalizing the effective model of Eq. (9), we obtain the $\Delta$ dependence of the nodal lines, as shown in 
(a)

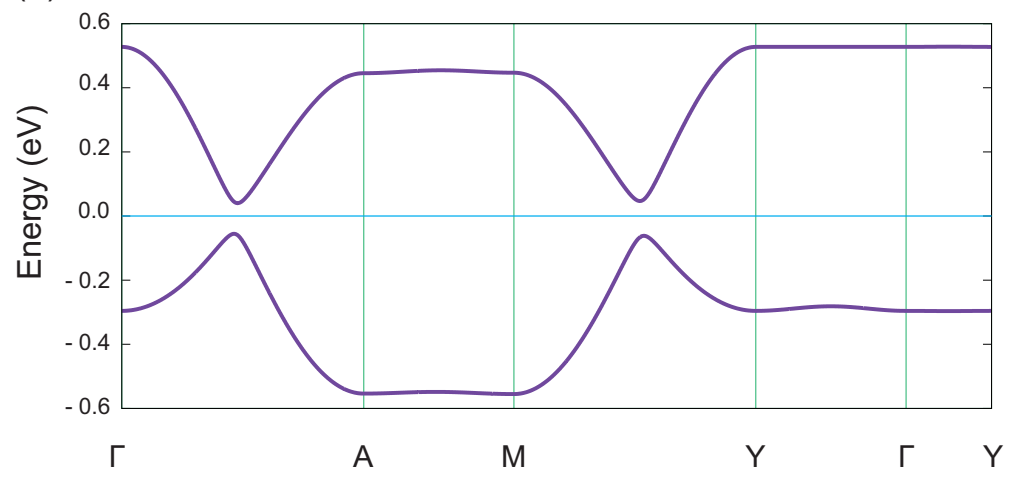

(b)

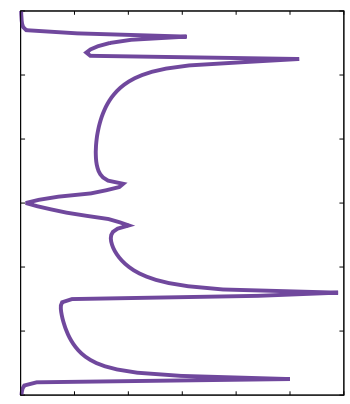

total DOS (c)

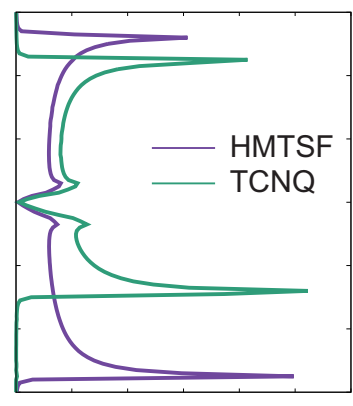

partial DOS

FIG. 5. (a) Energy dispersion and (b) the total DOS of the proposed tight-binding model. (c) Partial DOS for HMTSF and TCNQ.

(a)

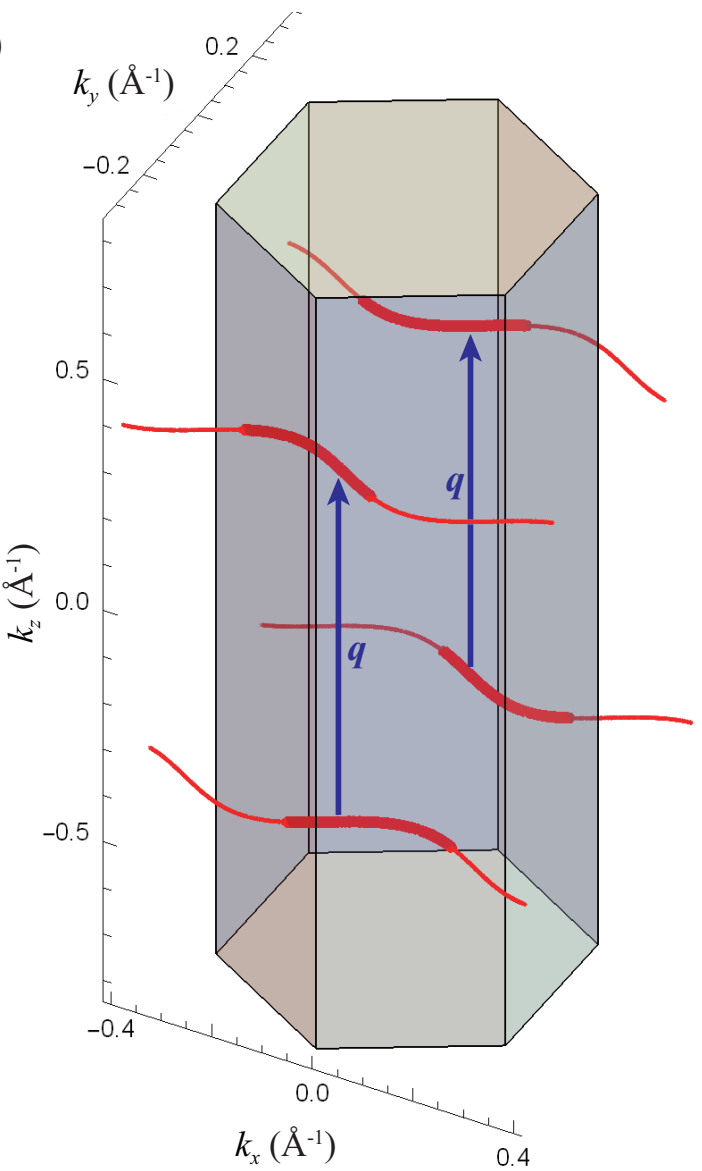

(b) $k_{y}\left(\AA^{-1}\right)$

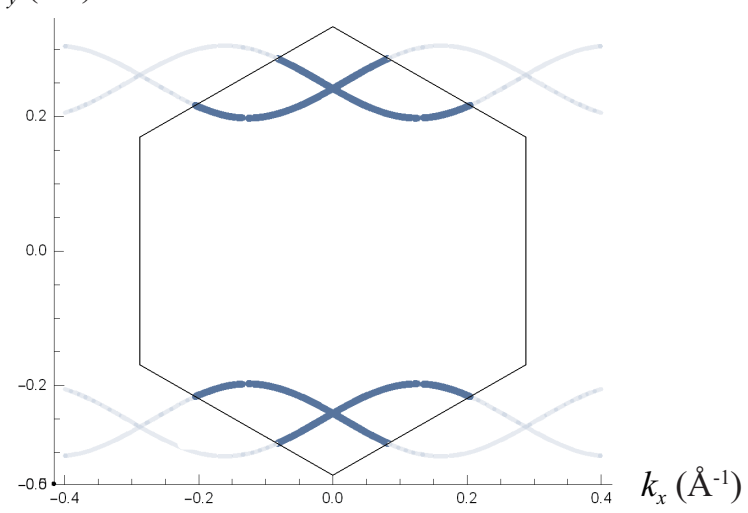

(c)

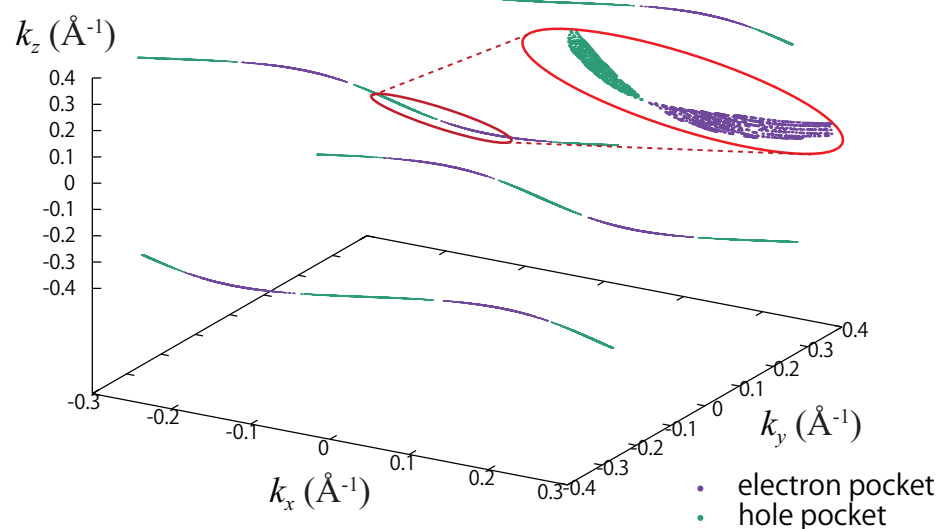

FIG. 6. (a) Configuration of the nodal lines in the $\boldsymbol{k}$ space. The nodal lines in (out of) the first Brillouin zone are indicated by thick (thin) lines. The nodal lines have $C_{2 y}$ rotation, inversion, and $\sigma_{y}$ mirror symmetry. The vector $\boldsymbol{q}$ represents the CDW nesting vector to be considered in Sec. V, which is on the $k_{x}=0$ plane. (b) Nodal lines projected onto the $k_{x}-k_{y}$ plane. They intersect on the $k_{x}=0$ line, owing to the $C_{2 y}$ rotational symmetry. (c) Fermi surface of HMTSF-TCNQ. Purple and green surfaces represent the electron and hole pockets, respectively. The Fermi surface consists of the alternating electron and hole pockets along the nodal lines. 
Fig. 7(a). The blue, orange, and green lines represent the nodal lines with $\Delta=5,10$, and $15 \mathrm{meV}$, respectively. For the case of $\Delta=0 \mathrm{meV}$ [Fig. 6(b)], the nodal lines touch each other at a point. However, with finite $\Delta$, a band gap opens at this point, which is connected by the nesting vector. As a result, closed nodal lines are formed. As $\Delta$ increases, the closed nodal lines become small and vanish when $\Delta \simeq 18 \mathrm{meV}$. Figures 7 (b) and $7(\mathrm{c})$ show the Fermi surfaces for the cases with $\Delta=5 \mathrm{meV}$ and $\Delta=10$ $\mathrm{meV}$, respectively. They consist of alternating electron and hole pockets along the nodal lines, as in the case of $\Delta=0 \mathrm{meV}$, and these CDW states are still semimetallic.

\section{PHYSICAL QUANTITIES}

In this section, we evaluate two physical quantities that are important to the experiments: the spin-lattice relaxation time $T_{1}$ and orbital magnetic susceptibility.

\section{A. Spin-lattice relaxation time $T_{1}$}

The spin-lattice relaxation time $T_{1}$ reflects the electronic state near the Fermi level. To experimentally confirm the existence of nodal lines, we theoretically evaluate $T_{1}$ for the normal phase. Although there are several types of the origins of spin-lattice relaxation, in this analysis, we focus on the Fermi contact term between the conduction electron and the nucleus in each molecule, which is given by [48-50],

$$
\frac{1}{\left(T_{1}\right)_{\alpha}} \propto T \int_{-\infty}^{\infty}\left[D_{\alpha}(\varepsilon)\right]^{2}\left(-f^{\prime}(\varepsilon)\right) d \varepsilon,
$$

where $\alpha$ indicates HMTSF or TCNQ, $D_{\alpha}(\varepsilon)$ is the DOS for the orbital $\alpha$, and $f^{\prime}(\varepsilon)$ is the derivative of the Fermi distribution function $f(\varepsilon)=\left[1+e^{\beta(\varepsilon-\mu)}\right]^{-1}$. We assume that the chemical potential does not change with temperature above $T_{c}$. Figure 8 shows the temperature dependence of $1 / T_{1}$ in the logscale without the CDW order parameter. The blue (orange) solid line represents the theoretical values evaluated by Eq. (10) for the HMTSF (TCNQ) molecule. The power $T^{3}$ is also shown for reference. We observe that $\left(T_{1}\right)_{\mathrm{HMTSF}}$ and $\left(T_{1}\right)_{\mathrm{TCNQ}}$ follow the power law $1 / T_{1} \propto T^{3.1}$, which is very close to the expected law $1 / T_{1} \propto T^{3}$ for pure two-dimensional Dirac electrons.

\section{B. Orbital magnetic susceptibility}

As mentioned in Sec. I, HMTSF-TCNQ shows a large diamagnetic susceptibility at low temperatures. To understand the origin and the temperature dependence, we evaluate the orbital magnetic susceptibility in the proposed model with nodal lines using the Fukuyama for- mula [29] given by,

$$
\chi_{\text {orbit }}=\frac{e^{2}}{\hbar^{2}} \sum_{n \boldsymbol{k}} \operatorname{Tr} \gamma_{x} \mathcal{G} \gamma_{y} \mathcal{G} \gamma_{x} \mathcal{G} \gamma_{y} \mathcal{G},
$$

where $\mathcal{G}$ represents the thermal Green's function

$$
\mathcal{G}=\left[i \varepsilon_{n}-H_{\mathrm{CDW}}(\boldsymbol{k})+\mu+i \Gamma \operatorname{sign}\left(\varepsilon_{n}\right)\right]^{-1},
$$

where $\varepsilon_{n}=(2 n+1) \pi k_{\mathrm{B}} T, \Gamma$, and $\mu$ are the Matsubara frequency, the damping rate of the electron, and the chemical potential, respectively. $\gamma_{i}$ is the current operator in the $i(=x$ or $y)$ direction, which is defined by $\gamma_{i}=\partial H_{\mathrm{CDW}}(\boldsymbol{k}) / \partial k_{i}$, and the $n$ summation means the sum over the Matsubara frequency $\varepsilon_{n}$. The chemical potential is determined by the charge-neutrality condition,

$$
\sum_{l \boldsymbol{k}} f\left(\varepsilon_{l \boldsymbol{k}}\right)=\sum_{l \boldsymbol{k}}\left[1-f\left(\varepsilon_{l k}\right)\right]
$$

where $\varepsilon_{l k}$ is the energy dispersion of the $l$ th band.

For simplicity, we assume the temperature dependence of the order parameter, as typically used for the BardeenCooper-Schrieffer superconductivity [51],

$$
\Delta(T)=\Delta_{0} \sqrt{1-\frac{T}{T_{c}}},
$$

where $\Delta_{0}$ is the order parameter at zero temperature. $T_{c}$ is set to $30 \mathrm{~K}$, which is in accordance with the experiment [52]. In the following analysis, we evaluate the chemical potential by only considering the temperature dependence through Eq. (14) and setting $\beta \rightarrow \infty$ in Eq. (13). This approximation is justified when $T \simeq 0$; however, we expect that it is still valid at high temperatures because the thermal fluctuation overpowers the error of this approximation.

Integrating Eq. (11) numerically, we obtain the temperature dependence of the orbital magnetic susceptibility as shown in Fig. 9(a) for $\Delta_{0}=10 \mathrm{meV}(\Gamma=0,3$, and 6 $\mathrm{meV}$ ). Above $T_{c}$, we observe that the orbital magnetic susceptibility increases negatively as the temperature decreases from the room temperature for every $\Gamma$. The nodal lines are the ensembles of Dirac electrons; therefore the Landau-Peierls contribution (the extension of the Landau diamagnetism to periodic systems) is expected to be very small $[26,53]$, and the diamagnetism is attributed to the interband effect $[22,23,26,27]$. Interband diamagnetism in two-dimensional Dirac electrons has a temperature dependence of $T^{-1}$ in the clean limit [54]. The inset of Fig. 9(a) indicates that the present numerical result for the $\Gamma=0 \mathrm{meV}$ case (shown in blue) asymptotically obeys the power law for the low temperatures $(\sim 30$ $\mathrm{K})$. For the region $T_{c}<T<100 \mathrm{~K}$, impurities affect the interband effect. Although the orbital magnetism in massive Dirac electron systems is not significantly sensitive to impurities [27], that in massless Dirac electrons is rather sensitive $[23,24]$.

Below $T_{c}$, we experience the effect of the nodal line deformation as well as the interband effect and impurity 
(a)

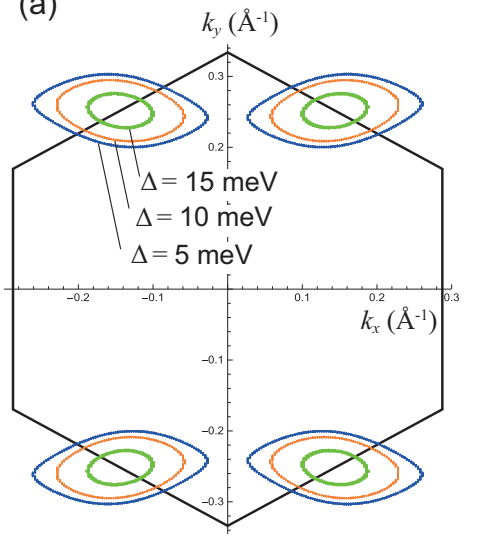

(b)

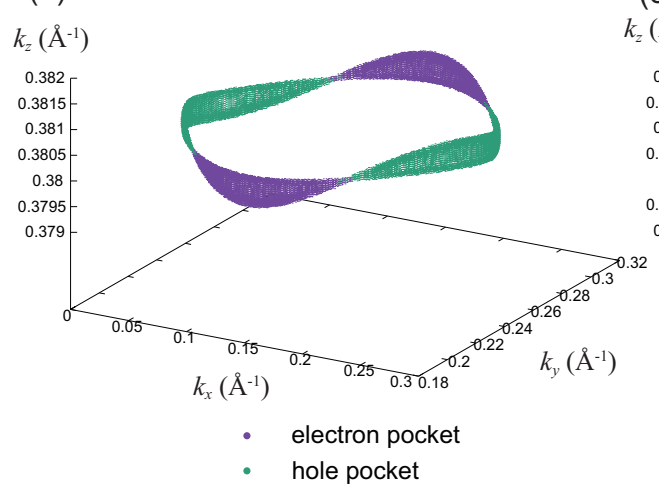

(c)

$k_{z}\left(\AA^{-1}\right)$

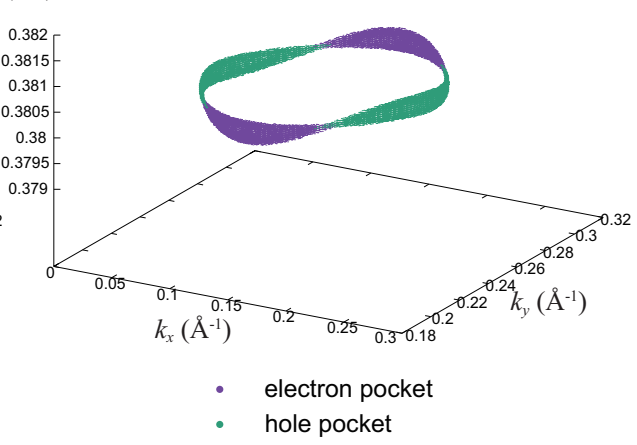

FIG. 7. (a) Projection of nodal lines onto the $k_{x}-k_{y}$ plane for the CDW phase. The blue, orange, and green lines correspond to $\Delta=5,10$, and $15 \mathrm{meV}$, respectively. Electron and hole pockets for (b) $\Delta=5 \mathrm{meV}$ and (c) $\Delta=10$ meV. The purple and green surfaces represent the electron and hole pockets, respectively.

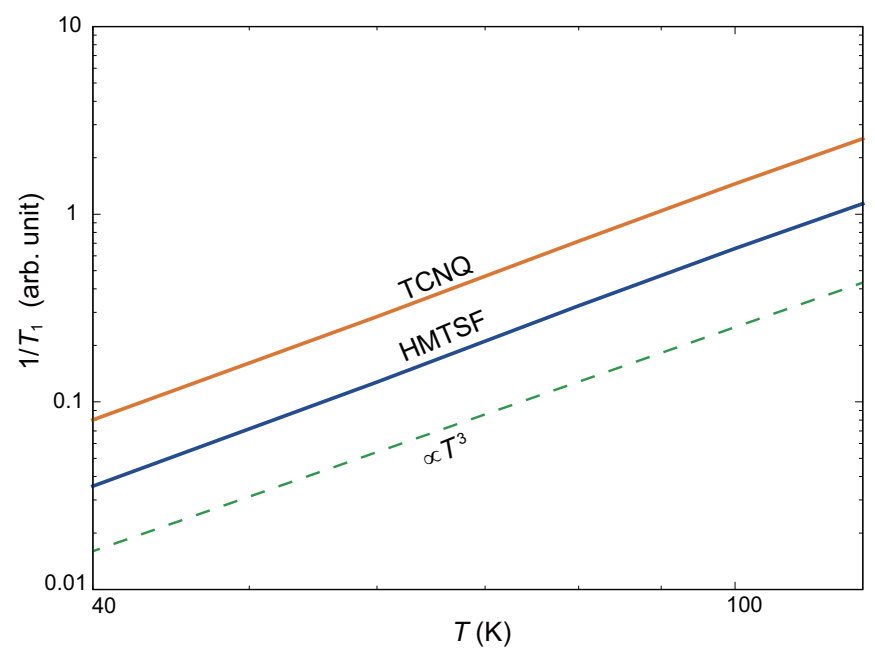

FIG. 8. Temperature dependence of $1 / T_{1}$ above $T_{c}$. The blue and orange lines represent the theoretical values for the HMTSF and TCNQ molecules, respectively. The powers for the two molecules are approximately 3.1. The power $T^{3}$ is also indicated by a dashed line for reference.

scattering. The inflection at $T_{c}$ is due to the order parameter. As the temperature decreases, the order parameter increases, and the nodal lines shrink, as discussed in the previous section. This implies a decrease in the number of the Dirac electrons, which results in suppression of diamagnetism. The relation between the magnitude of diamagnetism and the size of the nodal lines is discussed in detail in Ref. [55]. Note that a gap does not open in the DOS as long as $\Delta_{0}<18 \mathrm{meV}$, and massless Dirac electrons are present for the cases shown in Fig. 9(a). Therefore, the diamagnetism diverges at zero temperature for the case of $\Gamma=0 \mathrm{meV}$, whereas the divergence is suppressed with a finite $\Gamma$.
Figure 9(b) shows the orbital magnetic susceptibility for the cases of $\Delta_{0}=5,10$, and $15 \mathrm{meV}$, where we set $\Gamma=3 \mathrm{meV}$ as a typical value. For the case with a small $\Delta_{0}$, the diamagnetism monotonically increases as the temperature decreases. However, for the case with large $\Delta_{0}$, the diamagnetism is suppressed because the number of Dirac electrons decreases rapidly. Whether $\chi_{\text {orbit }}$ decreases or increases as the temperature decreases depends on the following three effects: the interband effect, impurity scattering, and nodal line deformation.

We compare the above theoretical results with a SQUID magnetometer measurement. The magnetizations were measured between 2-300 K using a SQUID magnetometer operated by a magnetic properties measurement system (Quantum Design Inc., CA, USA). A newly synthesized polycrystalline sample of $\sim 5 \mathrm{mg}$ is used. The core contribution of the diamagnetism estimated by Pascal's law $\left(-2.8 \times 10^{-4} \mathrm{emu} / \mathrm{mole}\right)$ is subtracted, as in the previous study [14]. The experimental data obtained in the cooling (heating) process are shown in Fig. 10 with circle (square) markers; the data reproduce the temperature dependence obtained in a previous study [14], including the absolute values.

Above $100 \mathrm{~K}$, the total magnetic susceptibility is paramagnetic. However, as the temperature decreases, the diamagnetism prevails, and finally reaches a plateau below the CDW phase transition temperature, $T_{c}=30 \mathrm{~K}$.

To understand the experimental results, we consider that the total magnetic susceptibility is given by

$$
\chi_{\mathrm{obs}}=a \chi_{\mathrm{orbit}}+b \chi_{\mathrm{Pauli}},
$$

with positive coefficients $a$ and $b$ and $\chi_{\text {Pauli }}$ is the Pauli paramagnetic contribution

$$
\chi_{\text {Pauli }}=-\mu_{\mathrm{B}}^{2} k_{\mathrm{B}} T \sum_{n \boldsymbol{k}} \operatorname{Tr} \mathcal{G}^{2},
$$

where $\mu_{\mathrm{B}}$ is the Bohr magneton $\mu_{\mathrm{B}}=|e| \hbar / 2 m$. The coefficient $a$ reflects the randomness of the polycrystal ori- 

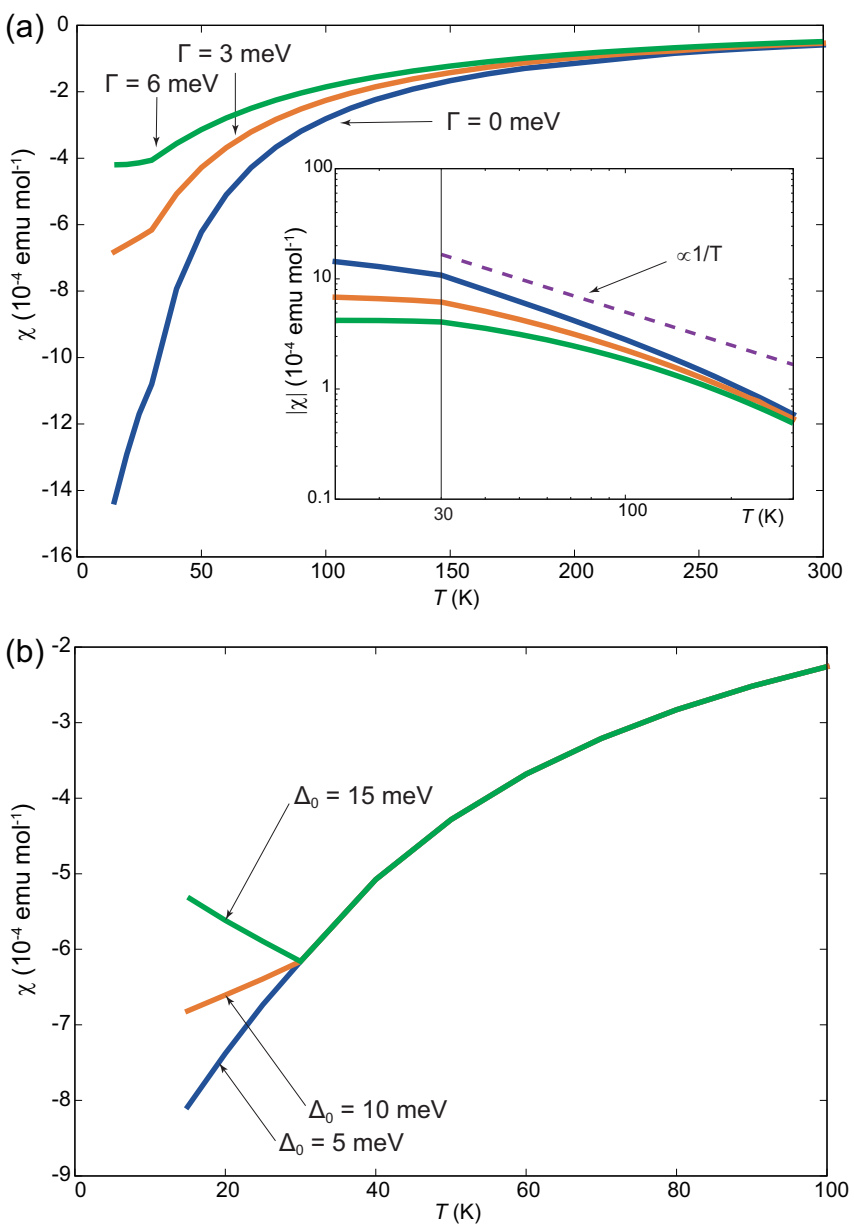

FIG. 9. (a) Orbital magnetic susceptibility for $\Delta_{0}=10 \mathrm{meV}$ as a function of the temperature. The blue, orange, and green lines correspond to $\Gamma=0 \mathrm{meV}, 3 \mathrm{meV}$, and $6 \mathrm{meV}$, respectively. Inset: Absolute value of the orbital magnetic susceptibility in the logscale. The power $T^{-1}$ is also shown for reference. (b) Orbital magnetic susceptibility for $\Gamma=3$ $\mathrm{meV}$ as a function of the temperature. The blue, orange, and green lines correspond to $\Delta_{0}=5 \mathrm{meV}, 10 \mathrm{meV}$, and $15 \mathrm{meV}$, respectively.

entation, and it is expected to be smaller than 1 because $\chi_{\text {orbit }}$ is calculated under a magnetic field in the direction of the largest diamagnetism. However, the coefficient $b$ ideally equals 1 . Note that $\chi_{\text {orbit }}\left(\chi_{\text {Pauli }}\right)$ is dominant at low (high) temperatures. Thus, the behavior below $T_{c}$ is primarily determined by $\chi_{\text {orbit }}$, which depends on $\Delta_{0}$ and $\Gamma$ up to a numerical factor. To reproduce the plateau that was experimentally observe, we choose $\Delta_{0}=11$ $\mathrm{meV}$ and $\Gamma=4 \mathrm{meV}$, and we set the numerical factor as $a=0.4$. However, for the $T>100 \mathrm{~K}$ region, the dominant contribution is from $b \chi_{\text {Pauli }}$, and to fit the data, we set $b=2.1$. We also show the obtained $a \chi_{\text {orbit }}, b \chi_{\text {Pauli }}$, and $\chi_{\text {obs }}$ in Fig. 10 with solid lines. The proposed theory is in excellent agreement with the experimental results for all temperature regions. This suggests that the ex-

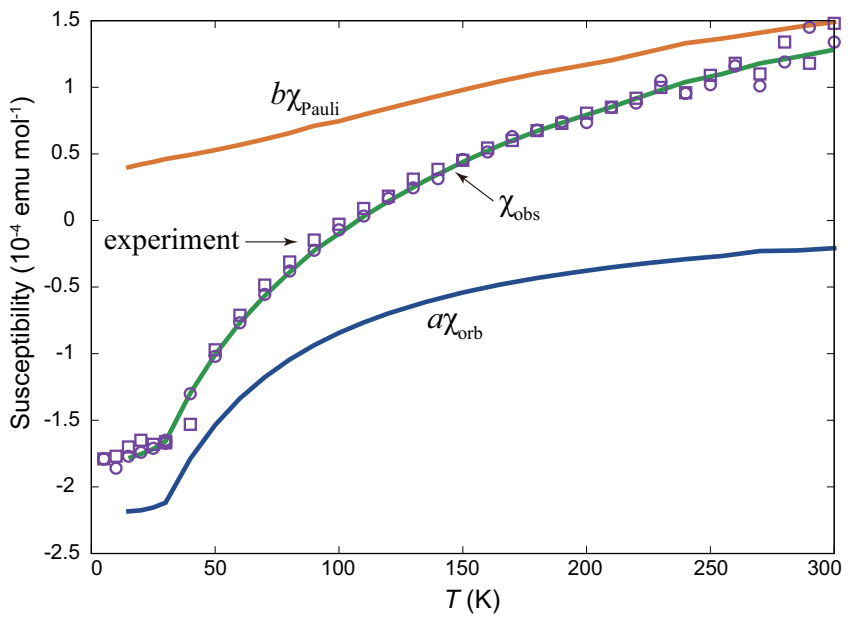

FIG. 10. Magnetic susceptibility as a function of temperature. The blue, orange, and green lines represent the theoretical evaluation of $a \chi_{\text {orbit }}, b \chi_{\text {Pauli }}$, and $\chi_{\mathrm{obs}}$, respectively, and the circle (square) markers represent the experimental data obtained by the SQUID magnetometer in the cooling (heating) process.

perimental result is explained by the above scenario, i.e., the anomalous large diamagnetism at low temperatures is explained by the conflict between the interband effect, impurity scattering, and the deformation of nodal lines.

It should be noted that the coefficient $b$ deviates from unity in this fitting. The origin of this deviation is not clear, however, it may be the intramolecular diamagnetism. Although this contribution has been partly subtracted as Pascal's law, a further consideration will be needed for a precise evaluation.

\section{CONCLUSION}

The present study is based on a first-principles calculation and tight-binding analysis, and it predicts that an organic complex HMTSF-TCNQ is a new candidate material for the nodal line semimetal. We have also clarified that the CDW deforms open nodal lines into closed ones. We evaluated the spin-lattice relaxation time $T_{1}$ and the magnetic susceptibility. An experiment was also conducted to investigate the magnetization, and the large anomalous diamagnetism, the origin of which had long been controversial, was reproduced. The present theoretical evaluation of magnetic susceptibility is in excellent agreement with the experimental results, and it has clarified that the conflict between the interband diamagnetism, impurity scattering, and nodal line deformation realizes this anomalous diamagnetism. Our evaluation of $T_{1}$ supports the existence of nodal lines, and it will be experimentally confirmed by means of a nuclear magnetic resonance measurement. This study is in progress, and the results will be presented elsewhere. 
For this material, many intriguing physical properties besides the magnetic susceptibility have been investigated, such as the Seebeck coefficient and Hall conductivity. These properties are also expected to be derived from the interplay between the CDW and nodal lines. These characteristics will be elaborated by the framework proposed in this study.

\section{ACKNOWLEDGMENTS}

We thank D. Miyafuji, T. Mizoguchi, M. Udagawa, H. Maebashi, and H. Fukuyama for fruitful discussions. We also thank R. Sugiura, T. Nakamura, and T. Takahashi for their magnetic susceptibility measurements, R. Kato for providing high quality samples, and H. Cui for providing crystal data. This work was supported by Grant-in-Aid for Scientific Research from the Japan Society for the Promotion of Science (Grand No. JP18H01162, JP19K03720, JP18K03482). S.O. and I.T. were supported by the Japan Society for Science Promotion (JSPS) through the Program for Leading Graduate Schools (MERIT).

\section{Appendix A: Wave functions and inversion parities at TRIM}

Figure 11 shows the wave functions for the valence and conduction bands at the TRIM, which are obtained by the first-principles calculation. For the $\Gamma$ point, the colors in the figure represent the signs of the wave functions. For other $\boldsymbol{k}$ points, the wave functions are generally complex and are shown in yellow. The cross-sections of the wave functions with the Brillouin zone boundary are shown in blue. Each wave function is the molecular orbital of either HMTSF or TCNQ. This suggests that the wave function at a generic point is also given by the linear combination of the wave functions for the two molecules, which justifies the assumption that the low-energy electronic state is described in terms of these two orbitals.

The parities of the inversion operation for the wave functions at TRIM are summarized in Table III. The symmetry indicator is calculated from these values for the valence band.

TABLE III. Parities of inversion operation for the wave functions at TRIM.

\begin{tabular}{lcccccc}
\hline \hline & $\Gamma$ & $\mathrm{V}$ & $\mathrm{Y}$ & $\mathrm{A}$ & $\mathrm{M}$ & $\mathrm{L}$ \\
\hline Valence & + & - & + & - & + & + \\
Conduction & - & - & - & - & + & - \\
\hline \hline
\end{tabular}

\section{Appendix B: Dirac cone}

The energy dispersion near a Dirac point when $k_{x}=$ 0 is shown in Fig. 12(a), and the contour plot of the difference of the energies $E_{+}-E_{-}$is shown in Fig. 12(b). We observed that the Dirac cone has strong anisotropy, and the system is two-dimensional only in the vicinity of the Dirac point.
[1] F. Qin, S. Li, Z. Z. Du, C. M. Wang, W. Zhang, D. Yu, H.-Z. Lu, and X. C. Xie, Theory for the charge-densitywave mechanism of $3 \mathrm{~d}$ quantum Hall effect, Phys. Rev. Lett. 125, 206601 (2020).

[2] J. Gooth, B. Bradlyn, S. Honnali, C. Schindler, N. Kumar, J. Noky, Y. Qi, C. Shekhar, Y. Sun, Z. Wang, B. A. Bernevig, and C. Fleser, Axionic charge-density wave in the Weyl semimetal $\left(\mathrm{TaSe}_{4}\right)_{2} \mathrm{I}$, Nature 575, 315 (2019).

[3] A. Kobayashi, S. Katayama, Y. Suzumura, and H. Fukuyama, Massless fermions in organic conductor, J. Phys. Soc. Jpn. 76, 034711 (2007).

[4] A. Kobayashi, Y. Suzumura, and H. Fukuyama, Hall effect and orbital diamagnetism in zerogap state of molecular conductor $\alpha$-(BEDT-TTF $)_{2} \mathrm{I}_{3}$, J. Phys. Soc. Jpn. 77, 064718 (2008).

[5] Z. F. Wang, Z. Liu, and F. Liu, Quantum anomalous Hall effect in 2D organic topological insulators, Phys. Rev. Lett. 110, 196801 (2013).

[6] L. Z. Zhang, Z. F. Wang, B. Huang, B. Cui, Z. Wang, S. X. Du, H.-J. Gao, and F. Liu, Intrinsic two-dimensional organic topological insulators in metal-dicyanoanthracene lattices, Nano Letters 16, 2072 (2016).

[7] Z. Liu, H. Wang, Z. F. Wang, J. Yang, and F. Liu, Pressure-induced organic topological nodal-line semimetal in the three-dimensional molecular crystal $\operatorname{Pd}(\text { dddt })_{2}$, Phys. Rev. B 97, 155138 (2018).

[8] R. Kato, H. Cui, T. Tsumuraya, T. Miyazaki, and Y. Suzumura, Emergence of the Dirac electron system in a single-component molecular conductor under high pressure, J. Am. Chem. Soc. 139, 1770 (2017).

[9] R. Kato and Y. Suzumura, A tight-binding model of an ambient-pressure molecular Dirac electron system with open nodal lines, J. Phys. Soc. Jpn. 89, 044713 (2020).

[10] T. Kawamura, D. Ohki, B. Zhou, A. Kobayashi, and A. Kobayashi, Tight-binding model and electronic property of Dirac nodal line in single-component molecular conductor $\left[\mathrm{pt}(\mathrm{dmdt})_{2}\right]$, J. Phys. Soc. Jpn. 89, 074704 (2020).

[11] R. Kato, H. Cui, T. Minamidate, H. H.-M. Yeung, and Y. Suzumura, Electronic structure of a single-component molecular conductor $[\mathrm{Pd}(\mathrm{dddt}) 2](\mathrm{dddt}=5,6$-dihydro1,4-dithiin-2,3-dithiolate) under high pressure, J. Phys. Soc. Jpn. 89, 124706 (2020).

[12] A. N. Bloch, D. O. Cowan, K. Bechgaard, R. E. Pyle, R. H. Banks, and T. O. Poehler, Low-temperature metallic behavior and resistance minimum in a new quasi onedimensional organic conductor, Phys. Rev. Lett. 34, 1561 (1975). 
[13] K. Bechgaard, D. O. Cowan, and A. N. Bloch, Stabilization of the organic metallic state: The properties of two substituted tetraselenafulvalenes and their TCNQ salts, Molecular Crystals and Liquid Crystals 32, 227 (1976).

[14] G. Soda, D. Jèrome, M. Weger, K. Bechgaard, and E. Pedersen, Spin relaxation and magnetic susceptibility studies of HMTSF-TCNQ, Solid State Commun. 20, 107 (1976).

[15] M. Weger, A model for the electronic band structure of HMTSeF-TCNQ, Solid State Commun. 19, 1149 (1976).

[16] K. Murata, Y. Fukumoto, K. Yokogawa, R. Takaoka, W. Kang, J. S. Brooks, D. Graf, H. Yoshino, T. Sasaki, and R. Kato, Magnetic-field-induced phase transitions in the quasi-one-dimensional organic conductor HMTSFTCNQ, Low Temp. Phys. 40, 371 (2014).

[17] K. Murata, W. Kang, K. Masuda, T. Kuse, T. Sasaki, K. Yokogawa, H. Yoshino, J. S. Brooks, E. S. Choi, A. Kiswandhi, and R. Kato, Field-induced CDW in HMTSF-TCNQ, Physica B 407, 1927 (2012).

[18] K. Murata, K. Yokogawa, K. Kobayashi, K. Masuda, T. Sasaki, Y. Seno, N. Rani Tamilselvan, H. Yoshino, J. S. Brooks, D. Jrome, K. Bechgaard, M. Uruichi, K. Yakushi, Y. Nogami, and R. Kato, Field-induced successive phase transitions in the charge density wave organic conductor HMTSF-TCNQ, J. Phys. Soc. Jpn. 79, 103702 (2010).

[19] D. Jérome and H. J. Schulz, Organic conductors and superconductors, Advances in Physics 31:4, 299 (1982).

[20] D. Jérome, Organic conductors: from charge density wave TTFTCNQ to superconducting (TMTSF) ${ }_{2} \mathrm{PF}_{6}$, Chemical Reviews 104, 5565 (2004).

[21] T. E. Phillips, T. J. Kistenmacher, A. N. Bloch, and D. O. Cowan, J. Chem. Soc., Chem. Commun. 66, 334 (1976).

[22] H. Fukuyama and R. Kubo, Interband effects on magnetic susceptibility. II. diamagnetism of bismuth, J. Phys. Soc. Jpn. 28, 570 (1970).

[23] H. Fukuyama, Anomalous orbital magnetism and Hall effect of massless fermions in two dimension, J. Phys. Soc. Jpn. 76, 043711 (2007).

[24] M. Nakamura, Orbital magnetism and transport phenomena in two-dimensional Dirac fermions in a weak magnetic field, Phys. Rev. B 76, 113301 (2007).

[25] M. Koshino and T. Ando, Anomalous orbital magnetism in Dirac-electron systems: Role of pseudospin paramagnetism, Phys. Rev. B 81, 195431 (2010).

[26] A. Raoux, F. Piéchon, J. N. Fuchs, and G. Montambaux, Orbital magnetism in coupled-bands models, Phys. Rev. B 91, 085120 (2015).

[27] Y. Fuseya, M. Ogata, and H. Fukuyama, Transport properties and diamagnetism of Dirac electrons in bismuth, J. Phys. Soc. Jpn. 84, 012001 (2015).

[28] S. Ozaki and M. Ogata, Universal quantization of the magnetic susceptibility jump at a topological phase transition, Phys. Rev. Research 3, 013058 (2021).

[29] H. Fukuyama, Theory of orbital magnetism of Bloch electrons: Coulomb interactions, Prog. Theor. Phys. 45, 704 (1971).

[30] K. Momma and F. Izumi, Vesta 3 for three-dimensional visualization of crystal, volumetric and morphology data, J. Appl. Crystallogr. 44, 1272 (2011).

[31] T. Hahn, U. Shmueli, and J. W. Arthur, International Tables for Crystallography, Vol. 1 (Reidel, Dordrecht, 1983).

[32] H. Cui and R. Kato, private communication.
[33] P. Giannozzi, S. Baroni, N. Bonini, M. Calandra, R. Car, C. Cavazzoni, D. Ceresoli, G. L. Chiarotti, M. Cococcioni, and I. D. et al., J. Phys. : Condens. Matter 21, 395502 (2009).

[34] P. Hohenberg and W. Kohn, Inhomogeneous electron gas, Phys. Rev. 136, B864 (1964).

[35] W. Kohn and L. J. Sham, Self-consistent equations including exchange and correlation effects, Phys. Rev. 140, A1133 (1965).

[36] J. P. Perdew, K. Burke, and M. Ernzerhof, Generalized gradient approximation made simple, Phys. Rev. Lett 77, 3865 (1996).

[37] L. Fu and C. L. Kane, Topological insulators with inversion symmetry, Phys. Rev. B 76, 045302 (2007).

[38] H. Po, A. Vishwanath, and H. Watanabe, Symmetrybased indicators of band topology in the 230 space groups, Nat. Commun. 8, 50 (2017).

[39] Z. Song, T. Zhang, Z. Fang, and C. Fang, Quantative mappings between symmetry and topology in solids, Nat. Commun. 9, 3530 (2018).

[40] Y. Kim, B. J. Wieder, C. L. Kane, and A. M. Rappe, Dirac line nodes in inversion-symmetric crystals, Phys. Rev. Lett. 115, 036806 (2015).

[41] Z. Song, T. Zhang, and C. Fang, Diagnosis for nonmagnetic topological semimetals in the absence of spin-orbital coupling, Phys. Rev. X 8, 031069 (2018).

[42] J. C. Slater and G. F. Koster, Simplified LCAO method for the periodic potential problem, Phys. Rev. 94, 1498 (1954).

[43] A. A. Mostofi, J. R. Yates, Y.-S. Lee, I. Souza, D. Vanderbilt, and N. Marzari, An updated version of wannier90: A tool for obtaining maximally-localised Wannier functions, Comput. Phys. Commun. 77, 3865 (2008).

[44] S. Ravy, P. Launois, R. Moret, and J.-P. Pouget, Case studies of molecular disorder, Z. Kristallogr. 220, 1059 (2005).

[45] H. Froöhlich, On the theory of superconductivity: the one-dimensional case, Proc. R. Soc. Lond. A 223, 296 (1954).

[46] C. G. Kuper, The thermal decomposition of ammonium perchlorate II. the kinetics of the decomposition, the effect of particle size, and discussion of results, Proc. R. Soc. Lond. A 227, 214 (1955).

[47] M. J. Rice and S. Strässler, Theory of a quasi-onedimensional band-conductor, Solid State Commun. 13, 125 (1973).

[48] T. Moriya, The effect of electron-electron interaction on the nuclear spin relaxation in metals, J. Phys. Soc. Jpn. 18, 516 (1963).

[49] Y. Suzumura, NMR relaxation rate of impure anisotropic quasi-one-dimensional superconductors, J. Phys. Soc. Jpn. 58, 2642 (1989).

[50] S. Katayama, A. Kobayashi, and Y. Suzumura, Electronic properties close to Dirac cone in two-dimensional organic conductor $\alpha$-(BEDT-TTF $)_{2} \mathrm{I}_{3}$, Eur. Phys. J. B 67, 139 (2009).

[51] A. L. Fetter and J. D. Walecka, Quantum theory of manyparticle systems (Dover, 2003).

[52] J. Cooper, M. Weger, D. Jrome, D. Lefur, K. Bechgaard, A. Bloch, and D. Cowan, Semi-metallic behaviour of HMTSF-TCNQ at low temperatures under pressure, Solid State Communications 19, 749 (1976).

[53] M. Ogata, Orbital magnetism of Bloch electrons: III. application to graphene, J. Phys. Soc. Jpn. 85, 104708 
(2016).

[54] J. W. McClure, Diamagnetism of graphite, Phys. Rev. 104, 666 (1956).
[55] I. Tateishi, V. Könye, H. Matsuura, and M. Ogata, Characteristic singular behaviors of nodal line materials emerging in orbital magnetic susceptibility and Hall conductivity, arXiv:2103.05591. 
$\Gamma$ point $\quad$ point $\quad Y$ point

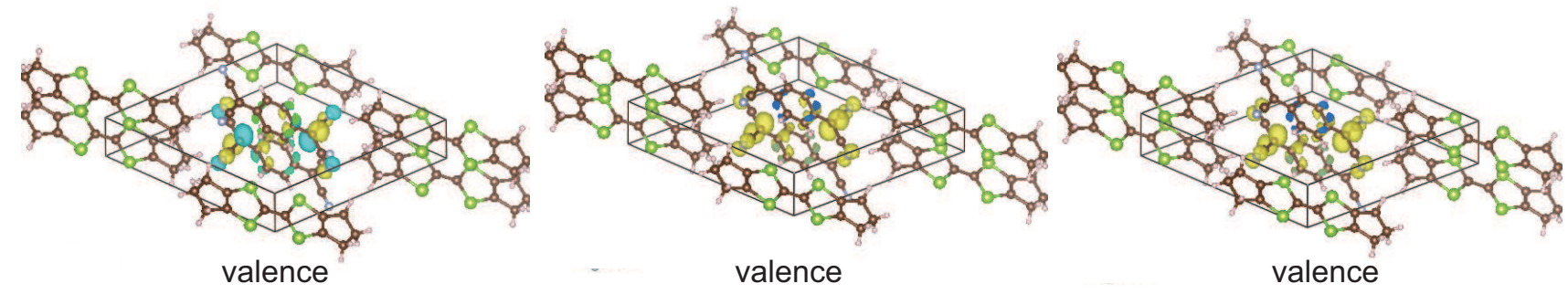

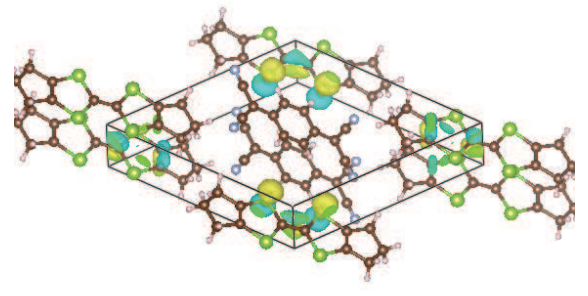

conduction

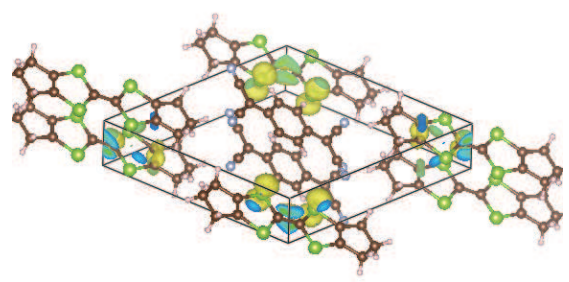

conduction

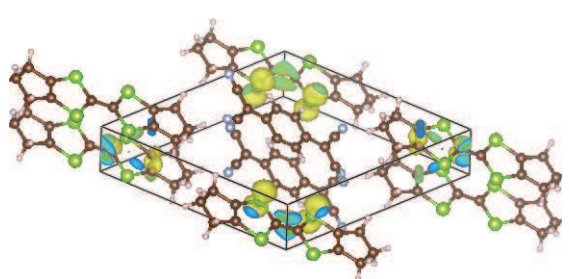

conduction

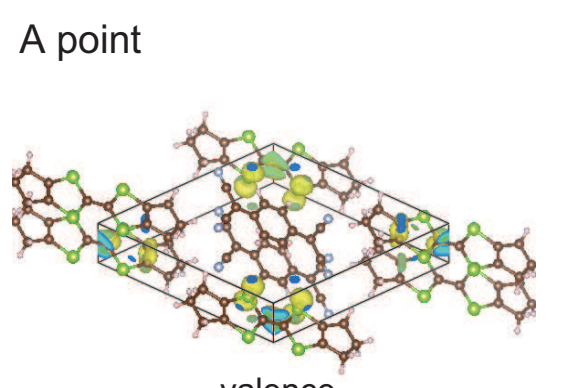

M point

$L$ point

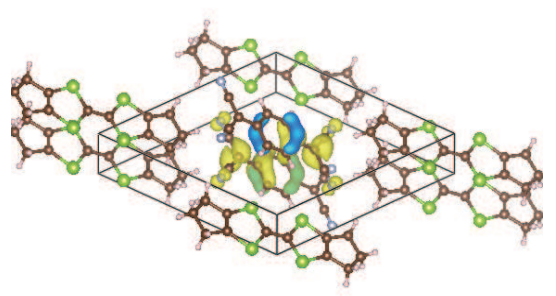

conduction
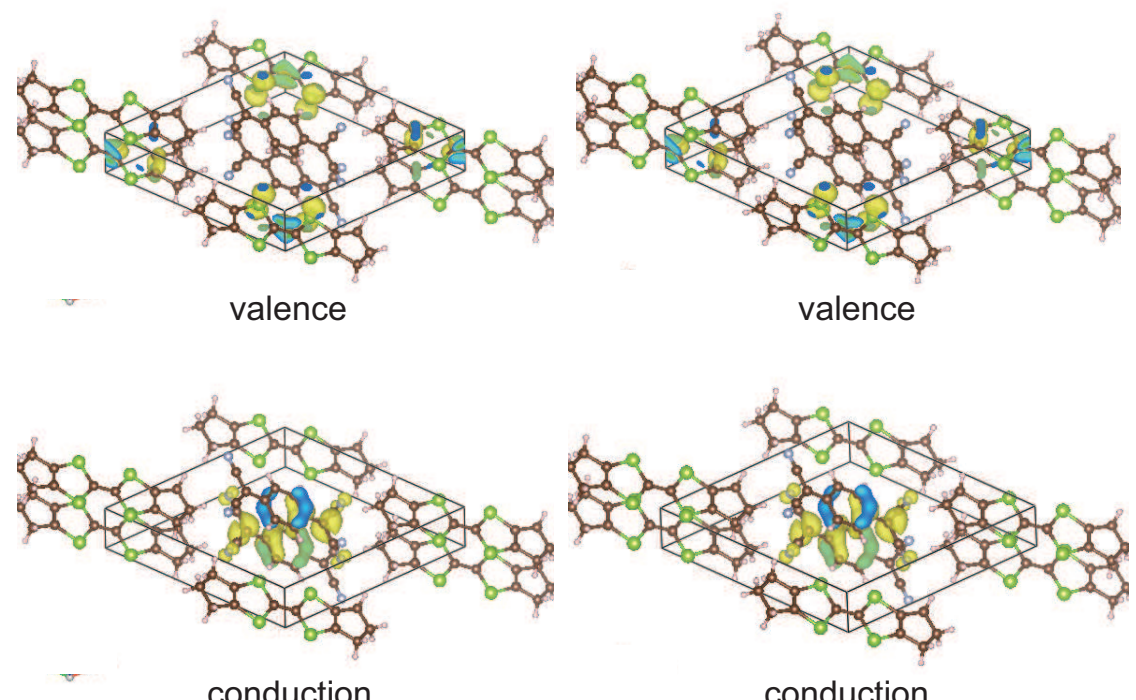

FIG. 11. Wave functions for the valence and conduction bands at the TRIM. 


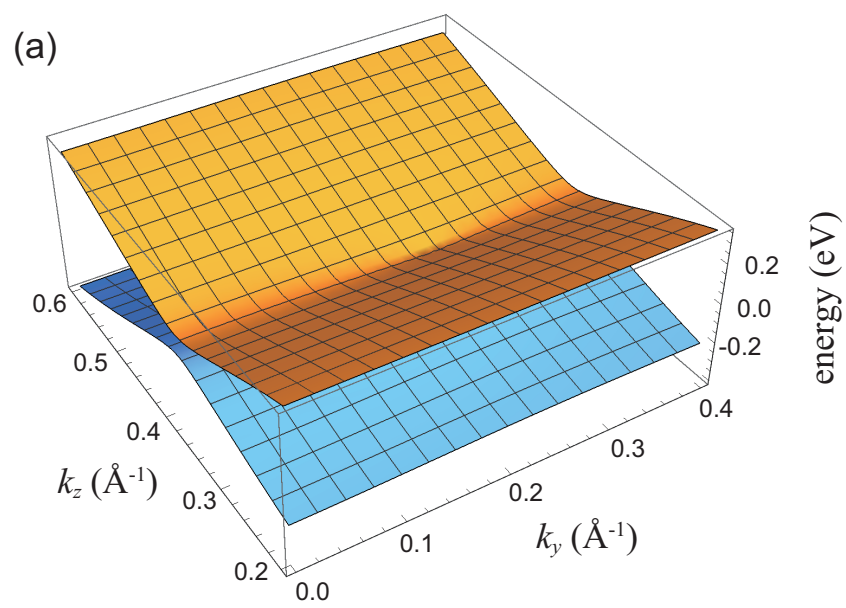

(b)

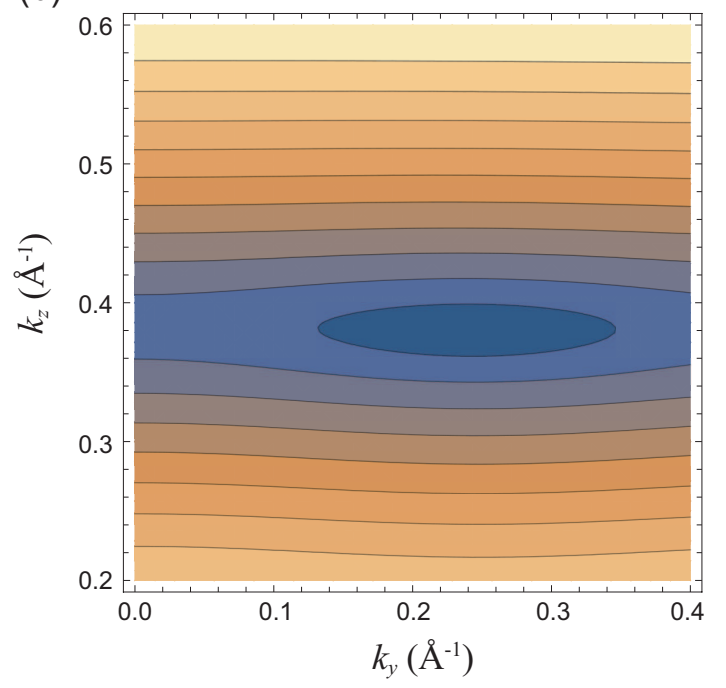

FIG. 12. (a) Energy dispersion near a Dirac point when $k_{x}=$ 0 . The orange (blue) surface corresponds to $E_{+}\left(E_{-}\right)$. (b) Contour plot of $E_{+}-E_{-}$. 